PROCEEDINGS OF THE

AMERICAN MATHEMATICAL SOCIETY

Volume 127, Number 2, February 1999, Pages 627-632

S 0002-9939(99)04925-4

\title{
ON SUSPENSIONS OF NONCONTRACTIBLE COMPACTA OF TRIVIAL SHAPE
}

\author{
UMED H. KARIMOV AND DUŠAN REPOVŠ \\ (Communicated by Ronald A. Fintushel) \\ Dedicated to the memory of the Teacher Hilol Karimov
}

\begin{abstract}
We prove that: (i) There exists a 2-dimensional noncontractible cohomologically locally connected cell-like compactum whose reduced suspension is a contractible ANR; (ii) If the suspension $\Sigma X$ of a compactum $X$ is contractible, then $X$ is weakly contractible.
\end{abstract}

\section{INTRODUCTION}

In 1904 Poincaré constructed the first example of a polyhedral homological 3sphere with nontrivial fundamental group [12]. The complement of an open star of a vertex in this space is a noncontractible acyclic finite polyhedron $P$. By the Mayer-Vietoris exact sequences and the Van Kampen Theorem it follows that the suspension $\Sigma P$ of this polyhedron is an acyclic space with the trivial fundamental group. It follows by the Hurewicz Theorem that therefore the suspension $\Sigma P$ has all homotopy groups trivial and is hence a contractible space. Complex $P$ is a noncontractible acyclic compactum. Every cell-like space is acyclic in Čech cohomology and every contractible compactum is clearly cell-like. So there is a natural question: Does there exist a noncontractible cell-like compactum whose suspension is contractible? [10, Problem 677]. The purpose of this paper is to prove the following related results:

Theorem (1.1). There exists a 2-dimensional noncontractible cohomologically locally connected cell-like compactum whose reduced suspension is a contractible ANR.

The example constructed in Theorem (1.1) is not weakly contractible and hence, according to Theorem (1.2) its unreduced suspension is not contractible.

Theorem (1.2). If the suspension $\Sigma X$ of a compactum $X$ is contractible, then $X$ is weakly contractible.

\section{Preliminaries}

To fix terminology we give some definitions. The suspension of a space $Z$, denoted by $\Sigma Z$, is defined as the quotient space of the product $Z \times I$ of $Z$ and the

Received by the editors June 17, 1996 and, in revised form, May 29, 1997.

1991 Mathematics Subject Classification. Primary 54C56, 55N05; Secondary 54D05, 55P40.

Key words and phrases. Čech cohomology, locally connected spaces, shape, suspension, contractibility.

(c)1999 American Mathematical Society 
segment $I=[0,1]$ in which $Z \times 0$ is identified to point $v_{0}$ and $Z \times 1$ is identified to another point $v_{1}$. Points $v_{0}$ and $v_{1}$ are called the vertices of $\Sigma Z$. The reduced suspension of space $Z$ relative to the point $z_{0} \in Z$, denoted by $\widetilde{\Sigma} Z\left(\operatorname{rel} z_{0}\right)$, is defined as the quotient space of $Z \times I$ in which $(Z \times 0) \cup\left(z_{0} \times I\right) \cup(Z \times I)$ is identified to a single point. For any point $(z, \tau) \in Z \times I$, we use $[z, \tau]$ to denote the corresponding point of $\Sigma Z$. For any map $f: Z^{\prime} \rightarrow Z$, the induced map $\Sigma f: \Sigma Z^{\prime} \rightarrow \Sigma Z$ is defined by $(\Sigma f)([z, \tau])=[f(z), \tau]$. The space $\Sigma Z$ is not always homotopically equivalent to $\widetilde{\Sigma} Z\left(\right.$ rel $\left.z_{0}\right)$. For example, let $Z$ be the one-point compactification $N^{*}$ of a countable discrete space. Then $\Sigma N^{*}$ and $\widetilde{\Sigma} N(\mathrm{rel} *)$ are not homotopically equivalent (they have countable and uncountable fundamental groups, respectively).

Space $Z$ is said to be weakly contractible (wc), if for every point $z_{0} \in Z$, there exists a neighborhood $V$ of the point $z_{0}$ such that the inclusion $V \subset Z$ is homotopically trivial. So every contractible or locally contractible space and every suspension is weakly contractible. To every mapping $g: Y \rightarrow \Sigma Z$ there is associated a canonical set-valued mapping $\phi_{g}: Y \rightarrow Z$ and a function $\psi_{g}: Y \rightarrow I$ defined by the equality $g(y)=\left[\phi_{g}(y), \psi_{g}(y)\right], y \in Y$. The mapping $g: \Sigma Z^{\prime} \rightarrow \Sigma Z$ is said to be flat if for every two points $\left[z_{1}^{\prime}, \tau_{1}^{\prime}\right]$ and $\left[z_{2}^{\prime}, \tau_{2}^{\prime}\right]$ with $\tau_{1}^{\prime}=\tau_{2}^{\prime}$, the following equality holds: $\psi_{g}\left(\left[z_{1}^{\prime}, \tau_{1}^{\prime}\right]\right)=\psi_{g}\left(\left[z_{1}^{\prime}, \tau_{2}^{\prime}\right]\right)$. Homotopy $H: \Sigma Z^{\prime} \times I \rightarrow \Sigma Z$ is called a flat homotopy if for every fixed $t_{0} \in I, H\left(, t_{0}\right): \Sigma Z^{\prime} \rightarrow \Sigma Z$ is a flat mapping.

Let $\mathcal{K}=\left\{K_{i-1} \stackrel{f_{i-1}}{\leftarrow} K_{i}\right\}_{i \in \mathbb{N}}$ be a sequence of compact polyhedron $K_{i}$, where $K_{0}$ is a point. Let $Y=\lim _{\overleftarrow{K}} \mathcal{K}$, and let $\left\{C\left(f_{0}, f_{1}, \ldots, f_{n}\right)\right\}_{n \in \mathbb{N}}$ be the associated (with $\mathcal{K}$ ) sequence of finite $\mathrm{CW}$-complexes. Now let $C\left(f_{0}, f_{1}, f_{2}, \ldots\right)$ be the infinite mapping cylinder (see, e.g. [13]) and let $Y_{\mathcal{K}}$ be the natural compactification of $C\left(f_{0}, f_{1}, f_{2}, \ldots\right)$ by $Y$, attached as a $Z$-set. The following is well known (see, e.g. $[3])$ :

Proposition (2.1). Space $Y_{\mathcal{K}}$ is an absolute retract.

Let $X_{\mathcal{K}}$ be the one-point compactification by some point $k$ of $C\left(f_{0}, f_{1}, f_{3}, \ldots\right)$. Obviously, $X_{\mathcal{K}}$ is homeomorphic to the quotient space $Y_{\mathcal{K}} / Y$. In the case when $Y$ is acyclic and $Y_{\mathcal{K}}$ is finite-dimensional it follows by the Whitehead theorem in shape theory [9] that $\operatorname{Sh}\left(X_{\mathcal{K}}\right)=\operatorname{Sh}\left(Y_{\mathcal{K}}\right)$. Therefore by Proposition (2.1) we have:

Proposition (2.2). Compactum $X_{\mathcal{K}}$ is a cell-like space.

Remark (2.3). If $\mathcal{K}^{\prime}$ is another inverse sequence for which $\operatorname{Sh} Y^{\prime}=\operatorname{Sh} Y$, then $X_{\mathcal{K}^{\prime}}$ is homotopically equivalent to $X_{\mathcal{K}}$ (see, e.g. [13, p. 375]).

\section{Proof of Theorem (1.1)}

Consider the Case-Chamberlin inverse sequence $\mathcal{P}[2]$ :

$$
P_{0} \stackrel{f_{0}}{\leftarrow} P_{1} \stackrel{f_{1}}{\leftarrow} P_{2} \stackrel{f_{2}}{\longleftarrow} \ldots
$$

where $P_{0}$ is a point, $P_{i}$ is a bouquet of two circles $S^{1} \vee S^{1}$, for every $i>0$, $f_{i}=f: S^{1} \vee S^{1} \rightarrow S^{1} \vee S^{1}$ is a continuous mapping which maps the natural generators $a$ and $b$ of the fundamental group $\pi_{1}\left(S^{1} \vee S^{1}\right)$ to the commutators $[a, b]$ and $\left[a^{2}, b^{2}\right]$ of $\pi_{1}\left(S^{1} \vee S^{1}\right)$, respectively. Since the inverse sequence $\mathcal{P}$ is acyclic and $\operatorname{dim} C\left(f_{0}, f_{1}, f_{2}, \ldots\right)=2$, we have by Proposition (2.2) the following:

Proposition (3.1). $X_{\mathcal{P}}$ is a 2-dimensional cell-like compactum. 
Next, we prove the following lemma.

Lemma (3.2). Let $X$ be a space with finitely generated Čech cohomology and suppose that for every point $x \in X$, there exists a basis of neighborhoods $\mathcal{U}_{x}=$ $\left\{U_{\alpha}\right\}_{\alpha \in A(x)}$ in $X$ such that for every $\alpha \in A(x)$, the boundary $\operatorname{Fr} U_{\alpha}$ also has finitely generated Čech cohomology. Then $X$ is a cohomologically locally connected space.

Proof. Consider the Mayer-Vietoris exact sequence:

$$
\cdots \rightarrow \check{H}^{n}(X) \rightarrow \check{H}^{n}\left(X \backslash U_{\alpha}\right) \oplus \check{H}^{n}\left(\bar{U}_{\alpha}\right) \rightarrow \check{H}^{n}\left(\operatorname{Fr} U_{\alpha}\right) \rightarrow \cdots .
$$

It follows that $\check{H}^{n}\left(\bar{U}_{\alpha}\right)$ is finitely generated. Now, by the continuity property of the Cech cohomology there exists a neighborhood $O_{\alpha} \subset U_{\alpha}$ such that the restriction $\check{H}^{n}\left(\bar{U}_{\alpha}\right) \rightarrow \check{H}^{n}\left(O_{\alpha}\right)$ is trivial (for $n=0$ consider the reduced cohomology). Therefore $X$ is indeed a clc space.

Condition that the Cech cohomology of $X$ is finitely generated is essential as the examples $X=\widetilde{\Sigma} N^{*}(\mathrm{rel} *)$ of $X=N^{*}$ show. From Lemma (3.2) and by construction of $X_{\mathcal{P}}$ we can deduce the following:

Proposition (3.3). Compactum $X_{\mathcal{P}}$ is a clc space.

In order to prove that $X$ is noncontractible we first need to prove the following two lemmas:

Lemma (3.4). For every $n>0$, the image of $a_{n} \in \pi_{1}\left(P_{n}\right)$ under the homomorphism $\pi_{1}\left(P_{n}\right) \stackrel{i_{n}}{\longrightarrow} \pi_{1}\left(X_{\mathcal{P}} \backslash\left\{P_{0}\right\}\right)$ is nonzero.

Proof. Let $Y_{n}$ be the quotient space of $X_{\mathcal{P}} \backslash P_{0}$ by $\overline{X_{\mathcal{P}} \backslash C\left(f_{0}, f_{1}, \ldots, f_{n}\right)}$ and let $j_{n}$ be the composition of the natural homomorphisms $\pi_{1}\left(P_{n}\right) \rightarrow \pi_{1}\left(X_{\mathcal{P}} \backslash P_{0}\right) \rightarrow \pi_{1}\left(Y_{n}\right)$. Clearly, $Y_{n}$ is homotopically equivalent to a finite $\mathrm{CW}$ complex whose fundamental group has the following presentation:

$$
\begin{gathered}
\left\langle a_{1}, b_{1}, a_{2}, b_{2}, \ldots, a_{n}, b_{n}: a_{2}\left[a_{1}, b_{1}\right], b_{2}\left[a_{1}^{2}, b_{1}^{2}\right], \ldots\right. \\
\left.\quad a_{n}\left[a_{n-1}, b_{n-1}\right], b_{n}\left[a_{n-1}^{2}, b_{n-1}^{2}\right],\left[a_{n}, b_{n}\right],\left[a_{n}^{2}, b_{n}^{2}\right]\right\rangle .
\end{gathered}
$$

By the Tietze transformations this presentation reduces to one with a single relation, $\left\langle a, b: \alpha_{n}(a, b)\right\rangle$. The words $\alpha_{n}(a, b)$ are defined inductively. Namely, $\alpha_{1}(a, b)=[a, b]$ and $\alpha_{k+1}(a, b)=\alpha_{k}\left([a, b],\left[a^{2}, b^{2}\right]\right), k \in \mathbb{N}$. In this presentation $j_{n}\left(a_{n}\right)$ equals $\alpha_{n-1}(a, b)$ and its weight is $n-1$ (cf. [7, 8]), hence $i_{n}\left(a_{n}\right) \neq 0$.

Lemma (3.5). Let $X$ be a compact space and suppose there is a point $x_{0} \in X$ such that $X \backslash\left\{x_{0}\right\}$ is locally contractible and there is a compact subset $P \subset X \backslash\left\{x_{0}\right\}$ such that no neighborhood $V \subset X \backslash P$ of $x_{0}$ is contractible in $X \backslash P$. Then $X$ is not a wc space.

Proof. Suppose to the contrary, that $X$ is a wc space. Let $V$ be a neighborhood of $x_{0}$ such that $i: V \hookrightarrow X$ is a contractible embedding. Any contraction which fixes $x_{0}$ will shrink sufficiently small neighborhood $W \subset V$ of the point $x_{0}$ in the complement of $P$. On the other hand, any contraction which moves $x_{0}$ will take a very small neighborhood of $x_{0}$ into a small neighborhood in $X \backslash\left(P \cup\left\{x_{0}\right\}\right)$ where it will shrink it in the complement of $P$. Contradiction. 
Proposition (3.6). The compactum $X_{\mathcal{P}}$ is not weakly contractible.

Proof. Following Lemma (3.5), let $X$ be $X_{\mathcal{P}}, x_{0}=p$ be the compactification point of the Case-Chamberlin infinite mapping cylinder, and $P=P_{0}$ the first term of the Case-Chamberlin inverse sequence. Let $V$ be any neighborhood of the point $p$ in $X_{\mathcal{P}} \backslash\left\{P_{0}\right\}$. Then by Lemma (3.4) there exists a loop in $V$ which is nontrivial in $X_{\mathcal{P}} \backslash$ $\left\{P_{0}\right\}$. Applying Lemma (3.5), we conclude that $X_{\mathcal{P}}$ is not weakly contractible.

Proposition (3.7). The reduced suspension $\widetilde{\Sigma} X_{\mathcal{P}}(\operatorname{rel} p)$ of $X_{\mathcal{P}}$ is an $A R$.

Proof. Consider the suspension $\Sigma \mathcal{P}$ of the Case-Chamberlin inverse sequence:

$$
\Sigma P_{0} \leftarrow \Sigma P_{1} \leftarrow \Sigma P_{1} \leftarrow \cdots
$$

and its inverse limit $\Sigma Y$. Obviously, $\widetilde{\Sigma} X_{\mathcal{P}}(\operatorname{rel} p)=Y_{\Sigma \mathcal{P}} / \Sigma Y$. By Proposition (2.1), $Y_{\Sigma \mathcal{P}}$ is an AR. By the Whitehead theorem [9], $\operatorname{Sh}(\Sigma Y)=\operatorname{Sh}(*)$. So $\widetilde{\Sigma} X_{\mathcal{P}}$ is a finite-dimensional cell-like image of the $\mathrm{AR}$ and therefore is also an $\mathrm{AR}[4,6]$.

Theorem (1.1) is now a direct consequence of Propositions (3.1), (3.3), (3.6), and (3.7).

\section{Proof of Theorem (1.2)}

Lemma (4.1). Let $Z^{\prime}$ and $Z$ be compact spaces, $\Sigma f: \Sigma Z^{\prime} \rightarrow \Sigma Z$ be a homotopically trivial flat mapping and $H: \Sigma Z^{\prime} \times I \rightarrow \Sigma Z$ a homotopy between $\Sigma f$ and a constant mapping. Suppose that for no $\tau^{\prime}, \tau \in I$, the set $\left\{H\left(\left[z^{\prime}, \tau^{\prime}\right], t\right) \mid z^{\prime} \in Z^{\prime}\right\}$ contains both vertices $v_{0}$ and $v_{1}$ of $\Sigma Z$. Then there exists a flat homotopy $H^{\prime}: \Sigma Z \times I \rightarrow \Sigma Z$ which connects $\Sigma f$ with the constant mapping.

Proof. Let $\phi_{H}$ and $\psi_{H}$ be the mappings corresponding to $H: \Sigma Z^{\prime} \times I \rightarrow \Sigma Z$ as defined in section 2. Fix the numbers $\tau^{\prime}$ and $t$. Let $a\left(\tau^{\prime}, t\right)$ and $b\left(\tau^{\prime}, t\right)$ be the minimum and the maximum of the function $\psi_{H}\left(\left[, \tau^{\prime}\right], t\right): Z^{\prime} \rightarrow I$. Define the mapping $H^{\prime}: \Sigma Z^{\prime} \times I \rightarrow \Sigma Z$ by the following formula:

$$
H^{\prime}\left(\left[z^{\prime}, \tau^{\prime}\right], t\right)=\left[\phi_{H}\left(\left[z^{\prime}, \tau^{\prime}\right], t\right), \frac{a\left(\tau^{\prime}, t\right)}{1+a\left(\tau^{\prime}, t\right)-b\left(\tau^{\prime}, t\right)}\right] .
$$

It is not difficult to check that $H^{\prime}$ is well-defined and that it has the required properties.

Let $H: \Sigma Z^{\prime} \times I \rightarrow \Sigma Z$ be a flat homotopy. Then there corresponds to $H$ a mapping $h: I^{2} \rightarrow I$ for which $h\left(\tau^{\prime}, t\right)=\psi_{H}\left(\left[z^{\prime}, \tau^{\prime}\right], t\right), z^{\prime} \in Z^{\prime}$. Let $p_{i}: I^{2} \rightarrow I$, $i=1,2$, be the projections, defined by $p_{1}(\tau, t)=\tau$ and $p_{2}(\tau, t)=t$, respectively.

Proof of Theorem (1.2). Suppose that $Z$ is a non-wc compactum at the point $z_{0} \in$ $Z$ and let $H: \Sigma Z \times I \rightarrow \Sigma Z$ be a homotopy between the identity mapping $\Sigma Z \rightarrow \Sigma Z$ and the constant one. Since $Z$ is a compact space there exists a number $\varepsilon>0$ such that no image by $H$ of any sets with diameter less than $\varepsilon$ contains both vertices $v_{0}$ and $v_{1}$.

Let $Z^{\prime}$ be a closed neighborhood of $z_{0}$ with diameter less than $\varepsilon$ and such that the inclusion $Z^{\prime} \hookrightarrow Z$ is homotopically nontrivial. Consider the inclusion $i: \Sigma Z^{\prime} \hookrightarrow \Sigma Z$. The restriction of $H$ onto $\Sigma Z^{\prime} \times I$ is a homotopy, connecting $i$ and the constant mapping. We can assume that the sets of points of $\Sigma Z^{\prime}$ with the same $\tau^{\prime}$ have diameter less than $\varepsilon$. Applying Lemma (4.1) we can assume also that $H$ is a flat homotopy. 
Let $A=h^{-1}(0)$ and $B=h^{-1}(1)$. We shall prove that then there exists a path $l:[0,1] \rightarrow I^{2}$ such that $l(0)=\left(\tau_{1}^{\prime}, 0\right), l(1) \in((0 \times I) \cup(I \times 1) \cup(1 \times I))$ and $\operatorname{Im} l \cap(A \cup B)=\varnothing$. Let $\delta$ be the distance between closed sets $A$ and $B$. Consider any natural triangulation $T$ of the square $I^{2}$ with mesh less than $\frac{\delta}{4}$. Let $P$ be the union of all simplexes of the $T$ intersecting $B$. The regular neighborhood $Q$ of polyhedron $P$ in $I^{2}$ is a submanifold of $I^{2}$ (see, e.g. [11, Proposition (3.10)]), not intersecting $A$. The boundary of manifold $Q$ is a discrete union of a finite number of simple closed curves. Denote by $S$ one of this curves which contains the vertex $(1,0)$ of $I^{2}$.

The curve $S$ contains vertices of $T$ which belong to $I \times 0$ or to $((0 \times I) \cup(I \times$ $1) \cup(1 \times I) \backslash\{(1,0)\})$. Denote all of them by $V_{0}$ and $V_{1}$ respectively. Vertices $V_{0}$ and $V_{1}$, divide $S$ into finite number of simple arcs. The number of arcs whose one end belongs to $V_{0}$ and other to $V_{1}$, is even. One of such arcs is a 1-simplex of $T$ with vertex $(1,0)$, which lies in $1 \times I$.

Therefore there exists the arc missing both $A$ and $B$, one end of which belongs to $V_{0}$ and other to $V_{1}$. Natural parametrisation of this arc gives the desired path $l:[0,1] \rightarrow I^{2}$.

Consider the cone $C\left(Z^{\prime}, \tau_{1}^{\prime}\right)=\left\{\left[z^{\prime}, \tau^{\prime}\right] \mid\left[z^{\prime}, \tau^{\prime}\right] \in \Sigma Z^{\prime}\right.$ and $\left.\tau^{\prime} \in\left[\tau_{1}^{\prime}, 1\right]\right\}$ and define a mapping $g: C\left(Z^{\prime}, \tau_{1}^{\prime}\right) \rightarrow \Sigma Z \backslash\left\{v_{0}, v_{1}\right\}$ by

$$
g\left(\left[z^{\prime}, \tau^{\prime}\right]\right)=H\left(\left[z^{\prime}, p_{1} l\left(\frac{\tau^{\prime}-\tau_{1}^{\prime}}{1-\tau_{1}^{\prime}}\right)\right], p_{2} l\left(\frac{\tau^{\prime}-\tau_{1}^{\prime}}{1-\tau_{1}^{\prime}}\right)\right) .
$$

Then because a cone is a contractible space, $g$ maps the base $\left[Z^{\prime}, \tau_{1}^{\prime}\right]$ inessentially to $\Sigma Z \backslash\left\{v_{0}, v_{1}\right\}$. However, from the homotopical point of view, the restriction of $g$ onto this base is the inclusion $Z^{\prime} \hookrightarrow Z$ which is homotopically nontrivial. Contradiction.

Corollary (4.2). The suspension $\Sigma X_{\mathcal{P}}$ is noncontractible.

Proof. By Proposition (3.6), the compactum $X_{\mathcal{P}}$ is not weakly contractible. So by Theorem (1.2), $\Sigma X_{\mathcal{P}}$ is noncontractible.

Example (4.3). Let for every $i \in \mathbb{N}, P_{i}$ be a finite noncontractible acyclic polyhedron. Let $Y=\bigvee_{i=1}^{\infty} P_{i}$ be the compact bouquet of $P_{i}$ 's. Although all the suspensions $\Sigma P_{i}$ are contractible spaces, $\Sigma Y$ is noncontractible, by Theorem (1.2).

Question (4.4). Is the double suspension $\Sigma\left(\Sigma X_{\mathcal{P}}\right)$ a noncontractible space?

Question (4.5). Does there exist a noncontractible locally contractible cell-like compactum?

\section{ACKNOWLEDGEMEnts}

The second author was supported in part by the Ministry of Science and Technology of the Republic of Slovenia grant No. J1-7039-0101-95. We wish to acknowledge the referee for several remarks and suggestions.

\section{REFERENCES}

1. G. E. Bredon, Sheaf Theory, (Second Edition), Springer-Verlag, New York, 1997. CMP 98:04

2. J. H. Case and R. E. Chamberlin, Characterization of tree-like continua, Pacific J. Math. 10 (1960), 73-84. MR 22:1868

3. R. J. Daverman and J. J. Walsh, Examples of cell-like maps that are not shape equivalences, Michigan Math. J. 30 (1983), 17-30. MR 85g:57008 
4. D. M. Hyman, ANR divisors and absolute neighborhood contractibility, Fund. Math. 62 (1968), 61-73. MR 37:4771

5. U. H. Karimov, An example of a space of trivial shape all of whose fine coverings are cyclic, Dokl. Akad. Nauk SSSR 286:3 (1986), 531-534 (in Russian); English transl. in Soviet Math. Dokl. 33 (1986), 113-117. MR 87i:55016

6. R. C. Lacher, Cell-like mappings, I, Pacific J. Math. 30 (1969), 717-731. MR 40:4941

7. R. C. Lyndon and P. E. Schupp, Combinatorial Group Theory, Springer-Verlag, Berlin, 1977. MR 58:28182

8. W. Magnus, A. Karrass and D. Solitar, Combinatorial Group Theory, Interscience, New York, 1966. MR 34:7617

9. S. Mardešić and J. Segal, Shape Theory: The Inverse System Approach, North-Holland, Amsterdam, 1982. MR 84b:55020

10. J. van Mill and G. M. Reed, Eds., Open Problems In Topology, North-Holland, Amsterdam, 1990. MR 92c:54001

11. C. P. Rourke and B. J. Sanderson, Introduction to Piecewise-Linear Topology, Ergebn. der Math. 69, Springer-Verlag, Berlin, 1972. MR 50:3236

12. H. Seifert and W. Threlfall, A Textbook of Topology, Pure Appl. Math. 89, Academic Press, New York, 1980. MR 82b:55001

13. L. C. Siebenmann, Chapman's classification of shapes. A proof using collapsing, Manuscripta Math. 16 (1975), 373-384. MR 55:4185

Institute of Mathematics, Tajik Academy of Sciences, Ul. Ainy 299, 734063 Dushanbe, TAJIKISTAN

E-mail address: umed@td.silk.org

Institute for Mathematics, Physics and Mechanics, University of Ljubljana, P. O. Box 2964, 1001 Luubluana, SLOvenia

E-mail address: dusan.repovs@fmf.uni-lj.si 